\title{
Exceso de proteínas en la pobreza: la paradoja del exceso de peso en niños colombianos
}

\author{
Excess protein in poverty settings: \\ the paradox of overweight \\ in colombian children
}

\begin{abstract}
The objective was to describe the total caloric value (TCV) derived from macronutrients in children under 18 years in relation to overweight in four Colombian cities between 2009-2011. Two 24 hour dietary recalls were measured in 505 children between 0 and 17 years, $51 \%$ living in urban areas. Nutritional status was calculated based on anthropometry. We used linear regression to test the association between TCV and excess weight. Overweight in children without stunting was $6.2 \%$. Overweight (BMI>=25) was $16.3 \%$ overall, with $18.2 \%$ overweight in urban areas and $14.2 \%$, in rural $(p=0.253)$. Children who were overweight usually consumed $0.25 \mathrm{~g} / \mathrm{kg} /$ day more protein (95\% Cl 0.11 to 0.40 ), $0.18 \mathrm{~g} / \mathrm{kg} /$ day less fat (95\%; -0.25 to -0.11$)$ and $1.80 \mathrm{~g} / \mathrm{kg} /$ day less carbohydrates (95\% Cl -2.50 to -1.10) compared to those without overweight, for all differences $p<0.0001$. Overweight subjects consumed $0.8 \%$ more protein derived TCV than those without excess weight, $p=0.006$. Being overweight was associated with TCV in Colombian children, particularly energy derived from protein consumption and also it was associated with the quantities/day of milk intake.
\end{abstract}

Key words Children; pediatric obesity; energy intake; diet; Colombia.

\section{INTRODUCCIÓN}

El exceso de peso y la obesidad están asociados al aumento de las enfermedades crónicas, tanto de las cardiovasculares como del cáncer (1). El exceso de peso se evidencia cada vez más a edades tempranas y la edad de inicio de ésta condición es un predictor del exceso y las enfermedades crónicas en la edad adulta (1). En los países de ingreso medio y bajo las cifras de exceso de peso siguen aumentando tanto en adultos como en niños (2). En Colombia el exceso de peso en adultos es mayor en población pobre y urbana, en niños de 5 y 17 años, la prevalencia fluctúa entre $8,7 \%$ a $16,1 \%$, ésta aumenta conforme aumenta el nivel de riqueza, el urbanismo, la seguridad alimentaria en el hogar (3), o cuando se abandona el patrón dietario "tradicional/almidón" (4).

El exceso de peso es determinado por condiciones biológicas, que ocurren en el individuo, culturales y sociales, que ocurren principalmente a nivel familiar, y por ambientales que

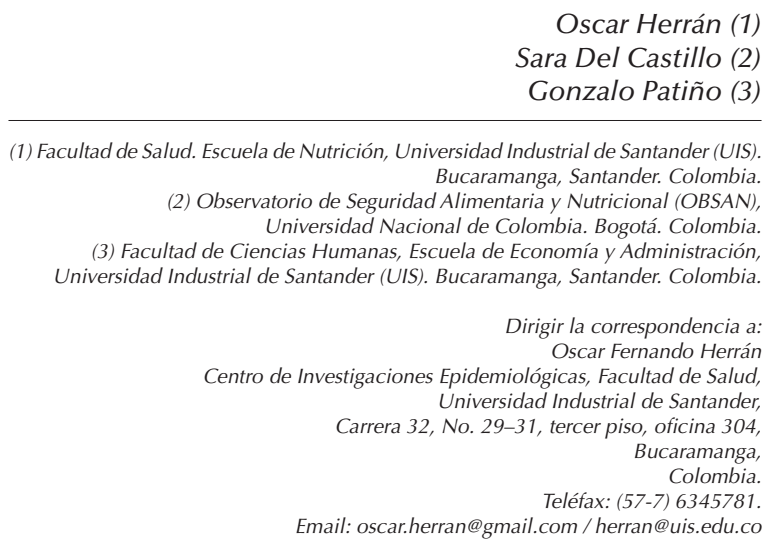

Este trabajo fue recibido el 25 de Abril de 2016 y aceptado para ser publicado el 16 de Diciembre de 2016.

ocurren a nivel de la microrregión $(3,5)$. La interacción de un número amplio de variables hace del exceso de peso y la obesidad asuntos complejos de abordar. En niños son muchas las explicaciones que se han dado como causales del exceso de peso. En edades tempranas el uso de fórmulas lácteas en reemplazo de la práctica de la lactancia (6), en preescolares y escolares por la inactividad física y el exceso de tiempo frente a pantallas, al exceso de consumo de energía, a la adopción de patrones dietarios ricos en bocadillos, también conocidos bajo el anglicismo de "snacks", bebidas azucaradas y tipo gaseosa, a los hábitos dietarios familiares (7), a las condiciones de salud mental y espirituales (8), al tipo de procesamiento de los alimentos (9), a los tipos y cantidades consumidas de alimentos entre sujetos con exceso y no, y de manera más general a la inequidad, la pobreza y la desigualdad $(3,4,10)$.

Las intervenciones con base en la dieta y la actividad física han sido las predominantes en el intento por controlar la 
pandemia, sin embargo, los resultados están lejos de lo esperado, las intervenciones se han centrado en la modificación de hábitos dietarios para evitar el consumo excesivo de energía, de grasas, bocadillos, bebidas azucaradas y tipo gaseosa y en la disminución del sedentarismo, éstas intervenciones por lo general se basan en evidencia lograda en contextos socioeconómicos y culturales diferentes a las de países en América Latina $(11,12)$.

A pesar de que la dieta es la fuente primaria de energía y la principal variable en muchas de las intervenciones para controlar el exceso de peso y la obesidad, son pocos los estudios que se aproximan a la ingesta usual en niños tanto a nivel individual como poblacional, dadas las complejidades técnicas, logísticas y los costos necesarios. La dieta es una compleja interacción de alimentos, que es susceptible de descomponerse en macro y micronutrientes y más allá, en la contribución relativa (\%) que hacen las principales fuentes dietarias, proteínas, grasas y carbohidratos a la energía total, descomposición técnicamente denominada valor calórico total (VCT). EI VCT es otra de las formas de aproximarse a la calidad de la dieta y sobre todo a las características de su composición (13).

Los objetivos de este estudio fueron describir el VCT en menores de 18 años y algunas variables biológicas, socioeconómicas y culturales en relación con el exceso de peso y además, establecer si existen diferencias en el tipo de alimentos y las cantidades consumidas de los mismos en niños con y sin exceso de peso.

\section{MÉTODOS}

Este estudio se clasifica como analítico a partir de datos repetidos sobre la ingesta dietaria de energía, (kilocalorías), proteínas, grasa total y carbohidratos y transversales del estado de nutrición, condiciones socioeconómicas, ambientales en menores de 18 años y de inseguridad alimentaria en sus hogares (INSA). Se desarrolló durante 2009-2011 en cuatro ciudades de Colombia, Suramérica. Bogotá es la capital del Colombia, Bucaramanga una ciudad intermedia y Tenjo y Sibaté municipios rurales articulados a Bogotá como proveedores de alimentos. Se realizó en ocho etapas a) cálculo de la muestra, b) selección de la muestra, c) fuentes de información, d) determinación del estado de nutrición y el exceso de peso, e) determinación del consumo dietario, f) determinación de la inseguridad alimentaria en el hogar (INSA), g) Aproximación al nivel socioeconómico de los menores y $h$ ) análisis estadístico.

\section{Cálculo de la muestra}

El estudio que dio origen a este análisis; "calidad de la dieta, percepción del hambre y seguridad alimentaria en población urbana y rural: estudio multicéntrico de evaluación de tecnología diagnóstica en Cundinamarca, Bogotá, Bucaramanga y su Área Metropolitana", obtuvo inicialmente una muestra de 450 hogares (270 urbanos y 180 rurales), con representatividad por estrato socioeconómico. Este tamaño de muestra permitió estimar una prevalencia mínima de percepción de hambre del 8,2\%, considerando un alfa de 0,05 , poder de $80 \%$ y un efecto del diseño de la muestra (DEFF) de 1,3. Complementariamente, 505 sujetos -los aquí analizados-, permitieron con un nivel de confianza del 95\%, una precisión absoluta de $4 \%$ y un DEFF de 1,3, estimar una prevalencia de exceso de peso del 15\%. Además, 505 sujetos permiten estimar una diferencia ente medias de $1 \%$ o más, con desviación estándar común de 4 , con datos de entrada de expuestos/no expuestos (\%); 13/12, un intervalo de confianza del $95 \%$ y poder del $80 \%$ (Epidata v. $4,1 \circledast$ ).

\section{Selección de la muestra}

El presente estudio limitó el análisis a los 505 menores de 18 años encuestados en el estudio "calidad de la dieta, percepción del hambre y seguridad alimentaria en población urbana y rural: estudio multicéntrico de evaluación de tecnología diagnóstica en Cundinamarca, Bogotá, Bucaramanga y su Área Metropolitana". En síntesis, en el área rural, dos veredas se seleccionaron al azar en cada municipio -Tenjo y Sibaté-, todos los hogares de esas veredas que aceptaron participar se encuestaron. Los hogares urbanos de Bogotá y Bucaramanga incluida su Área Metropolitana se seleccionaron por muestreo aleatorio estratificado en múltiples etapas. Las manzanas de las ciudades se clasificaron en seis estratos socioeconómicos de acuerdo con la metodología de la oficina de planeación municipal, aleatoriamente seis manzanas fueron seleccionadas en cada estrato y sus mapas actualizados, las viviendas en ellas se numeraron en orden consecutivo. En cada hogar se realizó un censo y todos sus integrantes se invitaron a participar.

\section{Fuentes de información}

Tres cuestionarios fueron aplicados a todos los menores. Un formato para recoger información sociodemográfica y biológica y dos recordatorios del consumo dietario de las últimas veinticuatro horas (R24H) (13). Los R24H fueron aplicados a todos los menores de manera repetida con intervalo mínimo de una semana para evitar respuestas con base en la memoria (13). La madre y/o el cuidador aportaron información junto con el menor sobre su consumo dietario.

\section{Determinación del estado de nutrición y el exceso de peso}

Todos los sujetos fueron pesados y tallados usando técnicas antropométricas estandarizadas. Los menores de 24 meses en posición decúbito supino, los demás de pie. El peso se tomó usando básculas electrónicas (SECA referencia 872, sensibilidad $50 \mathrm{~g}$ ) y la talla usando tallímetro y estadiómetros portátiles (SECA referencia 213, sensibilidad $1 \mathrm{~mm}$ y $5 \mathrm{~mm}$ respectivamente). El dato del peso y la talla fue el promedio de dos mediciones. Para obtener valores $Z$ o estandarizados frente a los de referencia los datos de los menores de 18 años fueron procesados en ANTRO® y ANTHRO PLUS®. El exceso de peso fue definido de acuerdo con los puntos de corte equivalentes para el IMC para el sexo y la edad; $>25$, siguiendo las recomendaciones del grupo internacional de trabajo en obesidad (IOTF) (14). El retraso para la talla (Stunting) fue establecido con base en puntajes $Z$ en el indicador Talla/Edad $(Z<2)$. La obesidad con base en puntajes $Z>2$ con el IMC $(\mathrm{kg} /$ $\mathrm{m} 2$ ), sin hacer diferencia entre menores o mayores de cinco años, utilizando el patrón de referencia de la Organización Mundial de la Salud.

La intensidad de la actividad física de los menores fue establecida por auto-reporte de ellos o de sus padres, con base en un formato simplificado que indagó por la ocupación principal, la realización, frecuencia y duración de actividades cotidianas; eg. En leve se clasificaron a los estudiantes y los oficinistas, en moderada a las personas que desarrollan actividades en el hogar, a los estudiantes que se desplazan caminando a su sitio de estudio, y en severa a los agricultores, obreros o amas de casa que además, realizan actividades de agricultura.

\section{Determinación del consumo dietario}

Los $\mathrm{R} 24 \mathrm{H}$ fueron traducidos a calorías y macro nutrientes mediante FoodCalc 1.3 (15) utilizando una tabla de alimentos compuesta de otras cuatro de amplio uso en Colombia, esta 
tabla agregada tiene 2284 ítems. El tamaño de la porción de alimentos se estableció mediante modelos abstractos validados previamente en las poblaciones estudiadas y ligados a la tabla de alimentos. El consumo usual de energía (kcal), proteínas (g), grasa total ( $\mathrm{g}$ ) y carbohidratos ( $\mathrm{g})$, fue establecido con base en los dos R24H utilizando los métodos desarrollados por la universidad de IOWA mediante PC-Side (16). Tres expresiones del consumo dietario fueron calculadas, a) Gramos usuales de macro nutrientes -proteínas, grasa y carbohidratos-, por kilogramo de peso y por día (g/kg/día), b) Distribución (\%) del $\mathrm{VCT}$, con base en la participación relativa del total de las kcal usuales derivadas del consumo de proteínas, grasas y carbohidratos, en la ingesta total de energía usual, con base en la siguiente ecuación; [(g usuales del macro nutriente*energía)/ total de kcal usuales] ${ }^{* 100}$. Para este cálculo se consideró que un $g$ de proteínas y de carbohidratos genera $4 \mathrm{kcal}$ y un $\mathrm{g}$ de grasa 9 kcal, y c) Tamaños de porción actual/día (g) de cada alimento identificado en los R24H.

\section{Determinación de la inseguridad alimentaria en el hogar (INSA).}

La inseguridad alimentaria se determinó a partir de una versión modificada y validada en Colombia del "Community Childhood Hunger Identification Project" y denominada ELCSA $(3,17)$. La ELCSA es una escala de quince ítems que contesta el jefe del hogar, en los hogares donde no hay menores de dieciocho años la escala se limita a diez ítems, todos dicotómicos, si la respuesta a los primeros cuatro ítems es negativa la ELCSA considera al hogar seguro y termina la aplicación de la misma $(3,17)$. La INSA se estableció en los 265 hogares donde utilizando los puntos de corte y algoritmos preestablecidos en la encuesta nacional de la situación nutricional en Colombia, 2010 (3).

Aproximación al nivel socioeconómico de los menores La clasificación socioeconómica de los menores se realizó con el Sistema de Identificación y Clasificación de Potenciales Beneficiarios para Programas Sociales (SISBEN), el cual permite identificar y clasificar a los hogares, familias y personas, conforme a sus condiciones de vida. Los niveles de SISBEN van desde el 1 hasta el 6, el nivel 1 es el más bajo en la escala socioeconómica y para ser beneficiario de programas estales hay que tener un nivel de 3 o inferior. En este estudio se utilizaron otras variables proximales del nivel socioeconómico, como el régimen de seguridad social en salud, y el nivel de INSA en el hogar.

\section{Análisis estadístico}

La descripción de las variables se realizó para las continuas con el promedio, su el error estándar (EE) y con el intervalo de confianza del 95\%. Para las discretas con el porcentaje (\%) y el intervalo de confianza del 95\%. Para las comparaciones entre grupos se utilizaron pruebas t de student, Ji2 y análisis de varianza según su nivel de medición. Las diferencias fueron ajustadas por los posibles confusores, tanto para el consumo usual expresado como VCT (\%), como para los tamaños de

\section{TABLA 1}

Valor calórico total (\%) a derivado del consumo usual/día de proteínas en menores de 18 años (sin embarazo) de acuerdo con condiciones sociodemográficas. Colombia, 2009-2011.

\begin{tabular}{|c|c|c|c|c|c|}
\hline Variable & $\mathrm{nb}$ & $\% \pm \mathrm{EEc}$ & $\begin{array}{c}\text { pd } \\
(I C ; 95 \%) e\end{array}$ & Diferencia ajustada & pf \\
\hline Todos & 505 & $12,0 \pm 0,11$ & & & \\
\hline Sexo & & & 0,239 & & 0,346 \\
\hline Masculino & 237 & $12,2 \pm 0,16$ & & $0,19(-0,21$ a 0,59$)$ & \\
\hline Femenino & 268 & $11,9 \pm 0,15$ & & Referencia & \\
\hline Edad (Años) & & & 0,053 & & 0,045 \\
\hline$<1$ & 33 & $10,4 \pm 0,51$ & & Referencia & \\
\hline 1 a 4 & 100 & $12,4 \pm 0,26$ & & $2,3(1,2$ a 3,5$)$ & \\
\hline 5 a 9 & 131 & $11,9 \pm 0,19$ & & $1,9(0,4$ a 3,5$)$ & \\
\hline 10 a 14 & 147 & $12,1 \pm 0,18$ & & $2,3(0,1 \mathrm{a}, 4,4)$ & \\
\hline 15 a 17 & 94 & $12,2 \pm 0,25$ & & $2,8(0,1$ a 5,5$)$ & \\
\hline Talla/Edad (Z score)g & & & $<0,0001$ & & 0,027 \\
\hline$<-2$ & 55 & $11,1 \pm 0,32$ & & $-0,6(-1,3$ a 0,0$)$ & \\
\hline$-2 a<-1$ & 146 & $11,9 \pm 0,20$ & & $-0,2(-0,6$ a 0,3$)$ & \\
\hline-1 a 1 & 255 & $12,3 \pm 0,14$ & & Referencia & \\
\hline$>1$ a 2 & 14 & $13,1 \pm 0,94$ & & $0,8(-0,4$ a 2,0$)$ & \\
\hline$>2$ & 6 & $13,8 \pm 0,93$ & & $0,3(-1,6$ a 2,1$)$ & \\
\hline IMC (Z score)g & & & 0,012 & & 0,027 \\
\hline$<-2$ & 11 & $11,9 \pm 0,92$ & & $-1,0(-2,4$ a 2,5$)$ & \\
\hline$-2 a<-1$ & 48 & $11,9 \pm 0,31$ & & $-0,6(-1,4$ a 0,2$)$ & \\
\hline-1 a 1 & 314 & $11,9 \pm 0,14$ & & Referencia & \\
\hline$>1$ a 2 & 69 & $12,5 \pm 0,28$ & & $0,3(-0,4$ a 1,0$)$ & \\
\hline$>2$ & 33 & $13,0 \pm 0,41$ & & $1,1(-0,0$ a 2,0$)$ & \\
\hline
\end{tabular}




\begin{tabular}{|c|c|c|c|c|c|}
\hline Exceso de peso (IMC-IOFT)h & & & $<0,0001$ & & 0,006 \\
\hline$<25$ & 360 & $12,0 \pm 0,12$ & & Referencia & \\
\hline $25+$ & 70 & $13,1 \pm 0,25$ & & $0,8(0,2$ a 1,3$)$ & \\
\hline Escolaridad & & & $<0,046$ & & 0,939 \\
\hline Preescolar o menos & 133 & $11,8 \pm 0,24$ & & Referencia & \\
\hline Hasta primaria & 170 & $12,1 \pm 0,17$ & & $-0,2(-0,9$ a 0,5$)$ & \\
\hline Hasta secundaria & 178 & $12,1 \pm 0,17$ & & $-0,6(-1,8$ a 0,5$)$ & \\
\hline Técnico/Universidad & 24 & $12,8 \pm 0,46$ & & $0,2(-1,3$ a 1,6$)$ & \\
\hline Actividad física & & & 0,024 & & 0,848 \\
\hline Leve & 380 & $11,9 \pm 0,13$ & & $0,5(-0,5$ a 0,6$)$ & \\
\hline Moderada/Severa & 125 & $12,5 \pm 0,19$ & & Referencia & \\
\hline Seguridad social & & & $<0,0001$ & & $<0,0001$ \\
\hline Subsidiado & 165 & $11,1 \pm 0,17$ & & Referencia & \\
\hline Contributivo & 264 & $12,4 \pm 0,14$ & & $0,7(0,2$ a 1,2$)$ & \\
\hline Vinculado & 45 & $12,0 \pm 0,35$ & & $0,6(-0,1$ a 1,4$)$ & \\
\hline Especial/Otro & 31 & $14,3 \pm 0,54$ & & $2,7(1,8$ a 3,6$)$ & \\
\hline Nivel de SISBEN & & & $<0,0001$ & & $<0,005$ \\
\hline 1 & 118 & $11,1 \pm 0,19$ & & Referencia & \\
\hline 2 & 181 & $11,7 \pm 0,16$ & & $0,5(-0,1$ a 1,9$)$ & \\
\hline $3 \circ 4$ & 30 & $12,6 \pm 0,51$ & & $1,2(0,2$ a 2,1$)$ & \\
\hline Resto & 176 & $12,9 \pm 0,19$ & & $0,9(0,3$ a 1,5$)$ & \\
\hline Recibe apoyo alimentario & & & $<0,0001$ & & 0,001 \\
\hline $\mathrm{Si}$ & 188 & $11,3 \pm 0,15$ & & Referencia & \\
\hline No & 317 & $12,6 \pm 0,14$ & & $0,7(0,3$ a 1,2$)$ & \\
\hline Consume alimentos fuera & & & $<0,009$ & & 0,228 \\
\hline $\mathrm{Si}$ & 170 & $12,4 \pm 0,19$ & & $0,3(-0,2$ a 0,7$)$ & \\
\hline No & 335 & $11,8 \pm 0,13$ & & Referencia & \\
\hline Inseguridad alimentaria en el hogari & & & $<0,0001$ & & $<0,0001$ \\
\hline No & 222 & $12,7 \pm 0,17$ & & Referencia & \\
\hline Leve & 196 & $11,6 \pm 0,16$ & & $-1,0(-1,4$ a $-0,6)$ & \\
\hline Moderada & 56 & $10,9 \pm 0,29$ & & $-1,7(-2,4 \mathrm{a}-1,1)$ & \\
\hline Severa & 24 & $11,8 \pm 0,41$ & & $-0,9(-1,8$ a 0,1$)$ & \\
\hline Área geográfica & & & $<0,0001$ & & $<0,0001$ \\
\hline Urbano & 258 & $12,7 \pm 0,14$ & & Referencia & \\
\hline Rural & 247 & $11,3 \pm 0,15$ & & $-0,8(-1,3$ a $-0,4)$ & \\
\hline Consumo usual kilocalorías & & & $<0,003$ & & 0,130 \\
\hline Q1 & 101 & $11,2 \pm 0,26$ & & Referencia & \\
\hline Q2 & 99 & $12,1 \pm 0,23$ & & $0,7(0,1$ a 1,4$)$ & \\
\hline Q3 & 100 & $12,2 \pm 0,24$ & & $0,7(0,1$ a 1,4$)$ & \\
\hline Q4 & 100 & $12,4 \pm 0,23$ & & $0,9(0,2$ a 1,5$)$ & \\
\hline Q5 & 99 & $12,3 \pm 0,23$ & & $0,5(-0,1$ a 1,2$)$ & \\
\hline Ciudad estudiada & & & $<0,0001$ & & 0,006 \\
\hline Bogotá & 177 & $12,7 \pm 0,19$ & & Referencia & \\
\hline Bucaramanga & 134 & $10,9 \pm 0,21$ & & $-0,7(-1,4$ a $-0,1)$ & \\
\hline Tenjo & 113 & $11,8 \pm 0,20$ & & $-0,9(-1,4$ a $-0,3)$ & \\
\hline Sibate & 81 & $12,7 \pm 0,22$ & & $-1,2(-1,8$ a $-0,6)$ & \\
\hline
\end{tabular}

a Valor calórico total; [(g de proteínas/usuales/día*4)/Ingesta usual total de kilocalorías]*100

b Los totales pueden ser $<505$ por valores perdidos.

c Porcentaje del valor calórico total y su error estándar (EE).

d Test de tendencia lineal para los predictores ordinales. Para el sexo, la actividad física, la seguridad social, recibir apoyo alimentario,

consumir alimentos fuera de la casa, el área geográfica y la ciudad estudiada, P es con base en análisis de varianza (ANOVA).

e Las diferencias fueron ajustadas en un modelo de regresión lineal, con el valor calórico total (\%) derivado del consumo usual/día de calorías derivadas de proteínas

como la variable dependiente y el sexo, la edad (continua), el IMC equivalente según IOFT (14), la ingesta de kilocalorías usuales (continua), la escolaridad,

el nivel de actividad física, la seguridad social, consumir alimentos fuera de la casa, el área (urbano/rural), y el nivel de inseguridad/seguridad alimentaria en el hogar (3), como explicatorias. Para las ciudades estudiadas el modelo no incluyo el área geográfica por su colinealidad.

f Test ajustado para tendencia lineal o ANOVA para los predictores ordinales o categóricos.

g Con base en el patrón de referencia del crecimiento de la OMS, calculados con Antro® y Antro Plus ${ }^{\circledR}$.

$\mathrm{h}$ Con base en los valores equivalentes para IMC según IOFT (14).

i Con base en una versión modificada de la ELCSA (Sin los ítems 9 y 13) (3). 
porción de los alimentos consumidos. Este ajuste fue realizado utilizando modelos de regresión lineal, con el valor calórico total (\%) derivado del consumo usual/día de calorías provenientes de proteínas, grasa o carbohidratos, como la variable dependiente y el sexo, la edad (continua), el IMC equivalente según IOFT, la ingesta de kilocalorías usuales (continua), la escolaridad, el nivel de actividad física, la seguridad social, consumir alimentos fuera de la casa, el área (urbano/rural), y el nivel de inseguridad/seguridad alimentaria en el hogar, como explicatorias. Para las ciudades estudiadas el modelo no incluyó el área geográfica por su colinealidad. Las diferencias en la cantidad de alimento consumido también fueron ajustadas en modelos de regresión lineal con el tamaño de la porción de un alimento especifico como variable dependiente y el exceso de peso (binomial), el sexo, la edad (continúa) y el nivel socioeconómico (SISBEN). Las correlaciones se establecieron con el coeficiente $r$ de Pearson, reportando su intervalo de confianza del 95\% (IC 95\%).

\section{Calidad de los datos}

Todas las encuestas, incluidos los R24H fueron realizadas por nutricionistas dietistas entrenados durante dos semanas en los métodos utilizados, el entrenamiento hizo énfasis en técnicas de fijación de la memoria y en cómo utilizar los modelos de alimentos previamente validados en la población blanco, para estimar el tamaño de la porción consumida. Las encuestas fueron revisadas diariamente por los encuestadores y semanalmente por supervisores de campo. La codificación de los alimentos en los $\mathrm{R} 24 \mathrm{H}$ se realizó el mismo día al terminar la entrevista con base en la descripción detallada del alimento o preparación. Los datos sociodemográficos fueron traducidos a "bits" con un lector óptico [Teleform], los de dieta, por digitadores. Todas las bases de datos fueron sometidas a doble digitación, procesos sucesivos de verificación y limpieza con algoritmos lógicos hasta asegurar que los datos se correspondían con los escritos. El procesamiento de datos junto con el análisis fue realizado con STATA 13.1 SE StataCorp, 2013 (18).

El estudio fue aprobado por el Comité de Ética en Investigación de la Facultad de Salud de la Universidad Industrial de Santander. Para todos los participantes se obtuvo un consentimiento informado por escrito de sus padres o representantes legales.

\section{RESULTADOS}

Todos los menores de 18 años del estudio que dio origen a este análisis fueron declarados elegibles, 505 sujetos fueron analizados, 237 son niños (46,9\%) y 268 niñas $(53,1 \%)$. La edad media fue de 9,3 años (IC 95\%; 8,9 a 9,8), sin diferencia por sexo $(p=0,474)$.

Características de los niños encuestados

En total, fueron encuestados $33(6,5 \%)$ menores de un año, 100 (19,8\%) entre uno y cuatro años, 131 (26,0\%) entre 5 y 9 años, 147 (29,1\%) entre 10 y 14 años y 94 (18,6\%) entre 15 y 17 años. El $75,3 \%$ fue clasificado como con actividad física leve, $23,0 \%$ con moderada y $1,7 \%$ con actividad física severa,

TABLA 2

Valor calórico total (\%) a derivado del consumo usual/día de grasa en menores de 18 años (sin embarazo) de acuerdo con condiciones sociodemográficas. Colombia, 2009-2011.

\begin{tabular}{|c|c|c|c|c|c|}
\hline Variable & $\mathrm{nb}$ & $\% \pm \mathrm{EEC}$ & $\begin{array}{c}\text { pd } \\
\text { (IC; 95\%)e }\end{array}$ & Diferencia ajustada & pf \\
\hline Todos & 505 & $28,0 \pm 0,3$ & & & \\
\hline Sexo & & & 0,133 & & 0,151 \\
\hline Masculino & 237 & $27,5 \pm 0,4$ & & $-0,8(-1,9$ a 0,3$)$ & \\
\hline Femenino & 268 & $28,4 \pm 0,4$ & & Referencia & \\
\hline Edad (Años) & & & $<0,0001$ & & 0,005 \\
\hline$<1$ & 33 & $38,0 \pm 1,4$ & & Referencia & \\
\hline 1 a 4 & 100 & $27,8 \pm 0,6$ & & $-11,3(-14,1 \mathrm{a}-8,4)$ & \\
\hline 5 a 9 & 131 & $26,8 \pm 0,5$ & & $-11,5(-15,4$ a $-7,7)$ & \\
\hline 10 a 14 & 147 & $27,7 \pm 0,5$ & & $-10,5(-16,0$ a $-5,0)$ & \\
\hline 15 a 17 & 94 & $26,6 \pm 0,6$ & & $-10,6(-17,6$ a $-3,7)$ & \\
\hline Talla/Edad (Z score)g & & & $<0,005$ & & 0,181 \\
\hline$<-2$ & 55 & $26,7 \pm 1,0$ & & $-0,7(-2,5$ a 1,1$)$ & \\
\hline$-2 a<-1$ & 146 & $26,8 \pm 0,5$ & & $-1,0(-2,3$ a 0,2$)$ & \\
\hline-1 a 1 & 255 & $28,6 \pm 0,4$ & & Referencia & \\
\hline$>1$ a 2 & 14 & $31,2 \pm 2,3$ & & $2,2(-1,0$ a 5,4$)$ & \\
\hline$>26$ & $28,7 \pm 2,2$ & & & $-2,7(-7,7$ a 2,2$)$ & \\
\hline IMC (Z score)g & & & 0,482 & & 0,023 \\
\hline$<-2$ & 11 & $27,4 \pm 2,1$ & & $0,3(-3,5$ a 4,2$)$ & \\
\hline$-2 a<-1$ & 48 & $27,5 \pm 1,0$ & & $0,8(-1,3$ a 2,8$)$ & \\
\hline-1 a 1 & 314 & $27,9 \pm 0,4$ & & Referencia & \\
\hline$>1$ a 2 & 69 & $28,0 \pm 0,7$ & & $-2,7(-4,6$ a $-0,8)$ & \\
\hline$>2$ & 33 & $28,7 \pm 1,0$ & & $-3,6(-6,6 a-0,7)$ & \\
\hline
\end{tabular}




\begin{tabular}{|c|c|c|c|c|c|}
\hline Exceso de peso $(\text { IMC-IOFT })^{\mathrm{h}}$ & & & $<0,043$ & & 0,524 \\
\hline$<25$ & 360 & $27,0 \pm 0,3$ & & Referencia & \\
\hline $25+$ & 70 & $28,5 \pm 0,6$ & & $0,5(-1,0$ a 1,9$)$ & \\
\hline Escolaridad & & & $<0,062$ & & 0,007 \\
\hline Preescolar o menos & 133 & $30,1 \pm 0,7$ & & Referencia & \\
\hline Hasta primaria & 170 & $26,7 \pm 0,4$ & & $0,2(-1,8$ a 2,1$)$ & \\
\hline Hasta secundaria & 178 & $27,5 \pm 0,5$ & & $3,9(0,8$ a 6,9$)$ & \\
\hline Técnico/Universidad & 24 & $28,2 \pm 1,2$ & & $4,6(0,7$ a 8,5$)$ & \\
\hline Actividad física & & & 0,056 & & 0,009 \\
\hline Leve & 380 & $27,6 \pm 0,3$ & & $-1,8(-3,2 a-, 4)$ & \\
\hline Moderada/Severa & 125 & $28,9 \pm 0,5$ & & Referencia & \\
\hline Seguridad social & & & $<0,002$ & & 0,009 \\
\hline Subsidiado & 165 & $26,2 \pm 0,5$ & & Referencia & \\
\hline Contributivo & 264 & $28,9 \pm 0,4$ & & $2,4(1,0$ a 3,7$)$ & \\
\hline Vinculado & 45 & $27,9 \pm 0,9$ & & $1,9(-0,1$ a 3,9$)$ & \\
\hline Especial/Otro & 31 & $29,6 \pm 1,1$ & & $2,6(0,2$ a 5,1$)$ & \\
\hline Nivel de SISBEN & & & $<0,0001$ & & $<0,0001$ \\
\hline 1 & 118 & $24,9 \pm 0,6$ & & Referencia & \\
\hline 2 & 181 & $27,1 \pm 0,5$ & & $1,6(0,1$ a 3,1$)$ & \\
\hline $3 \circ 4$ & 30 & $29,1 \pm 1,1$ & & $2,5(-0,0$ a 5,0$)$ & \\
\hline Resto & 176 & $30,7 \pm 0,5$ & & $4,5(2,9$ a 6,0$)$ & \\
\hline Recibe apoyo alimentario & & & $<0,0001$ & & $<0,0001$ \\
\hline $\mathrm{Si}$ & 188 & $25,7 \pm 0,4$ & & Referencia & \\
\hline No & 317 & $29,3 \pm 0,4$ & & $2,7(1,5$ a 3,9$)$ & \\
\hline Consume alimentos fuera & & & $<0,994$ & & 0,757 \\
\hline $\mathrm{Si}$ & 170 & $28,0 \pm 0,5$ & & $-0,2(-1,4$ a 1,0$)$ & \\
\hline No & 335 & $28,0 \pm 0,4$ & & Referencia & \\
\hline Inseguridad alimentaria en el hogari & & & $<0,0001$ & $<0,0001$ & \\
\hline No & 222 & $29,2 \pm 0,4$ & & Referencia & \\
\hline Leve & 196 & $27,3 \pm 0,5$ & & $-1,9(-3,1 \mathrm{a}-0,7)$ & \\
\hline Moderada & 56 & $26,8 \pm 1,0$ & & $-2,7(-4,5$ a $-0,9)$ & \\
\hline Severa & 24 & $23,4 \pm 1,3$ & & $-5,6(-8,1 \mathrm{a}-3,0)$ & \\
\hline Área geográfica & & & $<0,012$ & & 0,177 \\
\hline Urbano & 258 & $28,7 \pm 0,4$ & & Referencia & \\
\hline Rural & 247 & $27,2 \pm 0,4$ & & $-0,8(-2,1$ a 0,4$)$ & \\
\hline Consumo usual kilocalorías & & & $<0,003$ & & 0,084 \\
\hline Q1 & 101 & $29,3 \pm 0,9$ & & Referencia & \\
\hline Q2 & 99 & $26,4 \pm 0,6$ & & $-2,5(-4,2$ a $-0,8)$ & \\
\hline Q3 & 100 & $27,7 \pm 0,6$ & & $-1,7(-3,5$ a 0,0$)$ & \\
\hline Q4 & 100 & $28,9 \pm 0,5$ & & $-1,1(-2,9$ a 0,7$)$ & \\
\hline Q5 & 99 & $27,6 \pm 0,6$ & & $-2,5(-4,3 a-0,7)$ & \\
\hline Ciudad estudiada & & & $<0,002$ & 0,547 & \\
\hline Bogotá & 177 & $27,9 \pm 0,5$ & & Referencia & \\
\hline Bucaramanga & 134 & $30,4 \pm 0,7$ & & $1,1(-0,7$ a 2,8$)$ & \\
\hline Tenjo & 113 & $27,4 \pm 0,6$ & & $-0,6(-2,1$ a 0,9$)$ & \\
\hline Sibate & 81 & $27,1 \pm 0,6$ & $-0,6(-2,2$ a 1,0$)$ & & \\
\hline
\end{tabular}

a Valor calórico total; [(g de grasa/usuales/día*9)/Ingesta usual total de kilocalorías]*100

b Los totales pueden ser $<505$ por valores perdidos.

c Porcentaje del valor calórico total y su error estándar (EE).

d Test de tendencia lineal para los predictores ordinales. Para el sexo, la actividad física, la seguridad social, recibir apoyo alimentario,

consumir alimentos fuera de la casa, el área geográfica y la ciudad estudiada, P es con base en análisis de varianza (ANOVA).

e Las diferencias fueron ajustadas en un modelo de regresión lineal, con el valor calórico total (\%) derivado del consumo usual/día de calorías derivadas de proteínas como la variable dependiente y el sexo, la edad (continua), el IMC equivalente según IOFT (14), la ingesta de kilocalorías usuales (continua), la escolaridad,

el nivel de actividad física, la seguridad social, consumir alimentos fuera de la casa, el área (urbano/rural), y el nivel de inseguridad/seguridad alimentaria en el hogar (3), como explicatorias. Para las ciudades estudiadas el modelo no incluyo el área geográfica por su colinealidad.

f Test ajustado para tendencia lineal o ANOVA para los predictores ordinales o categóricos.

g Con base en el patrón de referencia del crecimiento de la OMS, calculados con Antro $®$ y Antro Plus

$\mathrm{h}$ Con base en los valores equivalentes para IMC según IOFT (14).

i Con base en una versión modificada de la ELCSA (Sin los ítems 9 y 13) (3). 
éstas condiciones no son diferenciales dado el sexo $(p=0,257)$. El 51,1\% provienen de la zona urbana, condición que no es diferencial por sexo $(p=0,710)$ o grupo etario, $(p=0,125)$. El régimen de salud que cubre la atención de estos niños fue subsidiado en $32,7 \%$, contributivo en $52,3 \%$, vinculado en $8,9 \%$, y el restante $6,1 \%$ en regímenes especiales, esta condición de cobertura de salud no es diferencial por sexo, $p=0,268$, pero si por zona de procedencia $(p<0,0001)$, en la zona rural predomina el régimen subsidiado con $51,4 \%$ y en la urbana el contributivo con $61,2 \%$. La clasificación socioeconómica predominante según el SISBEN en la zona rural fue el nivel $2(55,7 \%)$ y en la urbana el superior al nivel $4(54,3 \%),(p=$ $<0,0001)$. En la zona urbana el $45,7 \%$ de los niños consumen alimentos fuera del hogar, en la rural el 21,1\% ( $p<0,0001)$. El $29,5 \%$ de los hogares de niños urbanos recibe ayuda alimentaria al igual que el $45,3 \%$ de los rurales $(p<0,0001)$.

\section{Estado nutricional}

El retraso en talla ( $Z$ score $<-2$ ) en los niños fue $14,7 \%$ y en las niñas $8,7 \%(p=0,041)$, el retraso en talla no es diferencial dado los grupos etarios, mínimo 7,9\% en los niños entre 5 a 9 años y máximo $17,2 \%$ entre 1 y 4 años $(p=0,314)$. En la zona urbana el retraso es un $7,0 \%$ y en la rural $16,2 \%(p=0,002)$. La obesidad total según el IMC (Z score $>2)$ fue un 7,0\%, en los niños $8,9 \%$ y en las niñas $5,2 \%(p=0,012)$, la obesidad es diferencial dado los grupos etarios, mínimo 1,1\% en los niños entre 15 a 17 años y máximo 12,9\% entre 1 y 4 años $(p<0,0001)$. En la zona urbana la obesidad es de $7,9 \%$ y en la rural $6,0 \%, p=0,415$. La obesidad ( $Z$ score $>2$ ) es $12,1 \%$ en el nivel 1 del SISBEN, 45,5\% en el nivel 2, y de 33,3\% en el nivel superior al $4(p=0,316)$. El sobrepeso en niños sin retraso en talla es un $6,2 \%$. Según los puntos de corte equivalentes de IMC propuestos por IOFT el exceso de peso (IMC>= 25) es un $16,3 \%$, sin diferencia de por sexo $(p=0,942)$ o grupo etario $(p=0,256)$, pero si por nivel socioeconómico con base en el SISBEN, mínimo 3,9\% en el nivel 1 y máximo $21,1 \%$ en el nivel superior al $4(p=0,002)$. El exceso en la zona urbana es $18,2 \%$ y en la rural $14,2 \%(p=0,253)$. De los 505 hogares donde provienen estos niños, 283 cuentan con INSA, situación no diferencial dada la zona geográfica, urbana $48,0 \%$ y rural $41,0 \%(p=0,124)$.

\section{Consumo usual de energía (kcal/día) y macro nutrientes}

Los niños menores de 1 año consumen en promedio 996 kcal/día (IC 95\%; 850 a 1082), entre 1 y 4 años, 1981 kcal/ día (IC 95\%; 1881 a 2081), entre 5 y 9 años, 1941 kcal/día (IC 95\%; 1854 a 2028), entre 10 y 14 años, 1959 kcal/día (IC95\%; 1877 a 2041) y entre 15 y 17 años, 1918 kcal/día (IC 95\%; 1826 a 2010) ( $p<0,0001)$. El consumo de energía no es diferente por sexo, $(p=0,904)$ o nivel socioeconómico según el SISBEN $(p=0,665)$, pero si por la zona geográfica, $127 \mathrm{kcal} /$ día más en la urbana $(p=0,010)$. En un modelo ajustado por la edad, el sexo, el nivel socioeconómico, la zona geográfica y la ingesta de proteínas ( $\mathrm{g} / \mathrm{kg} / \mathrm{día})$, grasas $(\mathrm{g} / \mathrm{kg} /$ día) y carbohidratos ( $\mathrm{g} / \mathrm{kg} / \mathrm{día})$, se encontró que los niños con

TABLA 3

Valor calórico total (\%) a derivado del consumo usual/día de carbohidratos en menores de 18 años (sin embarazo) de acuerdo con condiciones sociodemográficas. Colombia, 2009-2011.

\begin{tabular}{|c|c|c|c|c|c|}
\hline Variable & $\mathrm{nb}$ & $\% \pm \mathrm{EEc}$ & $\begin{array}{c}\text { pd } \\
(I C ; 95 \%) e\end{array}$ & Diferencia ajustada & $\mathrm{pf}$ \\
\hline Todos & 505 & $60,0 \pm 0,3$ & & & \\
\hline Sexo & & & 0,343 & & 0,321 \\
\hline Masculino & 237 & $60,3 \pm 0,5$ & & $0,6(-0,6$ a 1,8$)$ & \\
\hline Femenino & 268 & $59,7 \pm 0,4$ & & Referencia & \\
\hline Edad (Años) & & & $<0,0001$ & & 0,059 \\
\hline$<1$ & 33 & $51,5 \pm 1,1$ & & Referencia & \\
\hline 1 a 4 & 100 & $59,8 \pm 0,7$ & & $9,0(5,7$ a 12,2$)$ & \\
\hline 5 a 9 & 131 & $61,3 \pm 0,6$ & & $9,6(5,2$ a 14,0$)$ & \\
\hline 10 a 14 & 147 & $60,2 \pm 0,6$ & & $8,2(2,0$ a 14,5$)$ & \\
\hline 15 a 17 & 94 & $61,2 \pm 0,7$ & & $7,8(-0,0$ a 15,7$)$ & \\
\hline Talla/Edad (Z score)g & & & $<0,0001$ & & 0,049 \\
\hline$<-2$ & 55 & $62,3 \pm 1,1$ & & $1,3(-7,0$ a 3,3$)$ & \\
\hline$-2 a<-1$ & 146 & $61,4 \pm 0,6$ & & $1,2(-1,0$ a 2,6$)$ & \\
\hline-1 a 1 & 255 & $59,1 \pm 0,4$ & & Referencia & \\
\hline$>1$ a 2 & 14 & $55,6 \pm 2,4$ & & $-3,0(-6,5$ a 0,5$)$ & \\
\hline$>2$ & 6 & $57,6 \pm 2,9$ & & $2,5(-2,9$ a 7,9$)$ & \\
\hline IMC (Z score)g & & & 0,228 & & 0,188 \\
\hline$<-2$ & 11 & $60,6 \pm 2,4$ & & $0,6(-3,6$ a 4,9$)$ & \\
\hline$-2 a<-1$ & 48 & $60,6 \pm 1,1$ & & $-0,2(-2,4$ a 2,1$)$ & \\
\hline-1 a 1 & 314 & $60,2 \pm 0,4$ & & Referencia & \\
\hline$>1$ a 2 & 69 & $59,6 \pm 0,8$ & & $2,4(0,3$ a 4,5$)$ & \\
\hline$>2$ & 33 & $58,3 \pm 1,2$ & & $2,6(-0,7$ a 5,8$)$ & \\
\hline
\end{tabular}




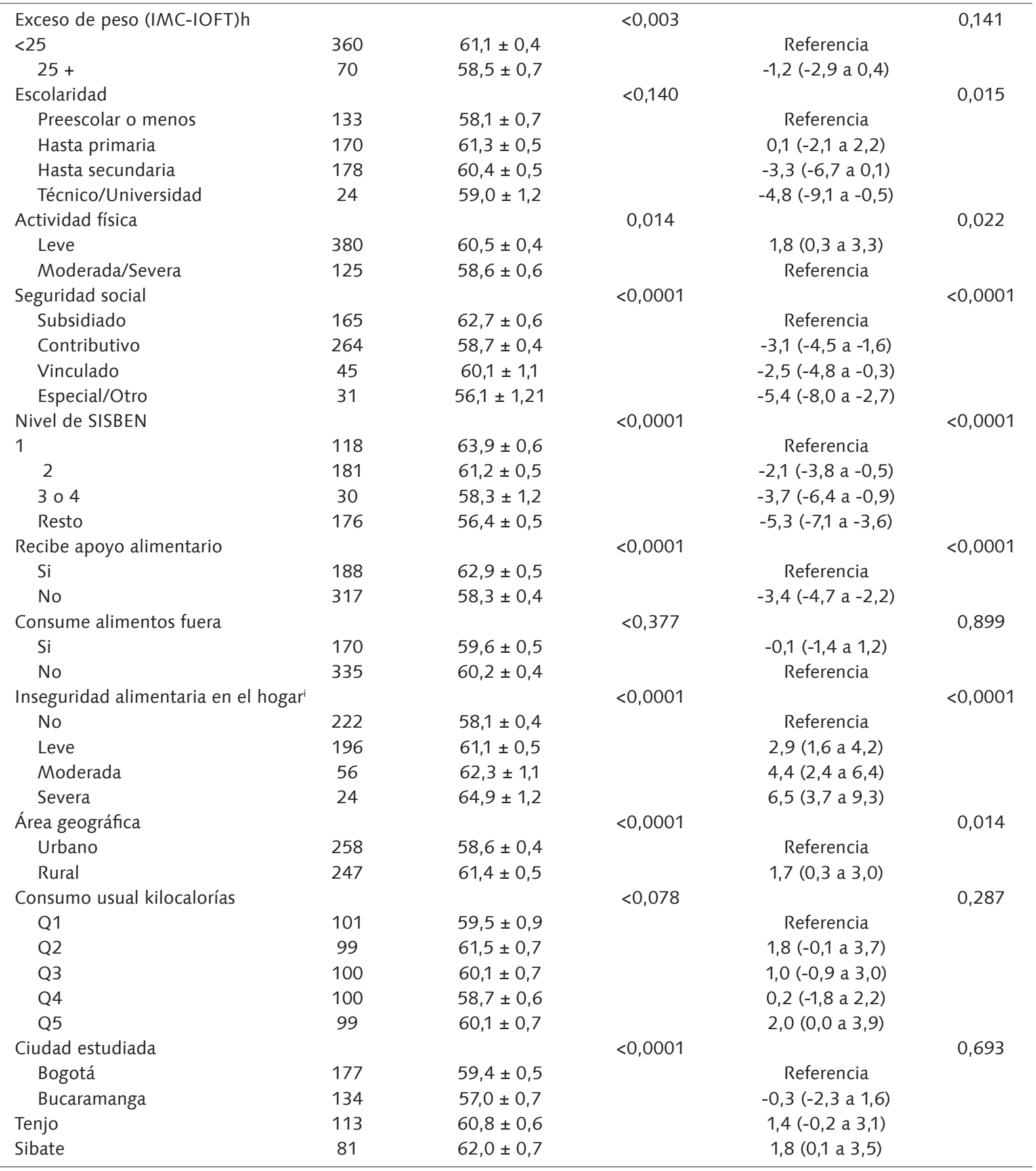

a Valor calórico total; [(g de carbohidratos/usuales/día*4)/Ingesta usual total de kilocalorías]*100

b Los totales pueden ser $<505$ por valores perdidos.

c Porcentaje del valor calórico total y su error estándar (EE).

d Test de tendencia lineal para los predictores ordinales. Para el sexo, la actividad física, la seguridad social, recibir apoyo alimentario,

consumir alimentos fuera de la casa, el área geográfica y la ciudad estudiada, $\mathrm{P}$ es con base en análisis de varianza (ANOVA).

e Las diferencias fueron ajustadas en un modelo de regresión lineal, con el valor calórico total (\%) derivado del consumo usual/día de calorías derivadas de proteínas

como la variable dependiente y el sexo, la edad (continua), el IMC equivalente según IOFT (14), la ingesta de kilocalorías usuales (continua), la escolaridad,

el nivel de actividad física, la seguridad social, consumir alimentos fuera de la casa, el área (urbano/rural), y el nivel de inseguridad/seguridad alimentaria en el hogar (3), como explicatorias. Para las ciudades estudiadas el modelo no incluyo el área geográfica por su colinealidad.

f Test ajustado para tendencia lineal o ANOVA para los predictores ordinales o categóricos.

g Con base en el patrón de referencia del crecimiento de la OMS, calculados con Antro® y Antro Plus

h Con base en los valores equivalentes para IMC según IOFT (14).

i Con base en una versión modificada de la ELCSA (Sin los ítems 9 y 13) (3). 
exceso de peso consumen usualmente $0,25 \mathrm{~g} / \mathrm{kg} /$ día más de proteínas (IC 95\%; 0,11 a 0,40), -0,18 g/ kg/día menos de grasa (IC 95\%; -0,25 a -0,11) y -1,80 g/kg/día menos de carbohidratos (IC 95\%; -2,50 a -1,10), que aquellos sin exceso de peso (para todas las diferencias $p<0,0001$ ). La figura 1, muestra el consumo de macro nutrientes por grupos de edad en niños con y sin exceso de peso.

\section{Distribución (\%) del valor calórico total (VCT)} con base en la ingesta usual/día

El aporte de $\mathrm{kcal}$ usual/día proveniente de proteínas es un $12,0 \%$ (IC 95\%; 11,8 a 12,4), el de grasas $28 \%$ (IC 95\%; 27,4 a 28,5) y el de carbohidratos 60\% (IC 95\%; 59,4 a 60,6). Los sujetos con exceso de peso consumen $0,8 \%$ más VCT derivado de proteínas que aquellos sin exceso $(p=0,006)$. La correlación entre el VCT derivado de proteínas y el VCT derivado de grasa es de $r=0,15$ (IC 95\%; 0,10 a 0,23), y con el VCT derivado de carbohidratos -0,47 (IC 95\%; -0,53 a -0,39). Las tablas 1 a 3 describen la relación del VCT con las variables socioeconómicas y biológicas.

Tamaños de porción y exceso de peso

Los sujetos reportaron su consumo en 909 R24H, en los que identificamos 672 alimentos, de ellos sólo en diez se evidenciaron diferencias en el tamaño de porción/día entre quienes tienen exceso de peso y no (tabla 4). Los sujetos con exceso de peso toman más leche líquida o reconstituida y más jugo de naranja, comen menos leguminosas (lenteja), salchichón (embutido de bajo costo) y gelatina además, toman menos agua de panela (es la bebida más popular entre colombianos, sin distingo de estrato socioeconómico, es preparada con agua y jugo concentrado y solidificado de caña de azúcar). $\mathrm{Ni}$ el consumo de bebidas gaseosas tipo Kola $(p=0,704)$, de bebidas azucaradas $u$ otro tipo de gaseosa no fue diferencial $(p=0,914)$. La proporción de consumo de leche líquida en el última día es un $36,5 \%$, la de gaseosas y refrescos azucarados $26,5 \%$ y la de bebidas tipo Kola 7,0\%.

\section{DISCUSIÓN}

Durante las dos últimas décadas en Colombia como en todos los países de la región, el sobrepeso y la obesidad en los niños han venido aumentando (2). Según la ENSIN-2010 en el grupo de 5-17 años la prevalencia de exceso de peso era $17,5 \%$ (3), similar a la aquí reportada. En la región, en niños de Argentina, Brasil, Chile, Estados Unidos y México, las cifras de exceso de peso están entre 15\% y 35\% (11, 19-23).

Nuestro análisis parte con la evidencia de que el consumo de energía en estos niños no es excesivo y que si bien está por encima de su requerimiento medio, cumple con la recomendación para garantizar su crecimiento adecuado. Sin embargo, el VCT derivado de proteínas en todos los grupos de edad es mayor que el deseable y asociado al exceso de peso. Ya ha sido reportado que en niños el consumo excesivo de proteínas está asociado al exceso, aumentando el riesgo de desórdenes metabólicos, los niveles de homocisteína y de factores de crecimiento como la insulina y por consiguiente, del riesgo de procesos ateroescleróticos $(6,24-26)$. Además en el largo plazo, los metabolitos del exceso de proteínas, pueden afectar el hígado y la función renal (24). Un estudio realizado en niños entre 10 y 12 años, encontró un VCT derivado de proteínas en el rango de $12 \%-14 \%$, que se correlacionó positivamente con el exceso de peso incluso, sin que estos menores cumplieran

\section{FIGURA 1}

Proteínas, grasas y carbohidratos (g/kg/día) en menores de 18 años y exceso de peso, Colombia, 2009-2011.

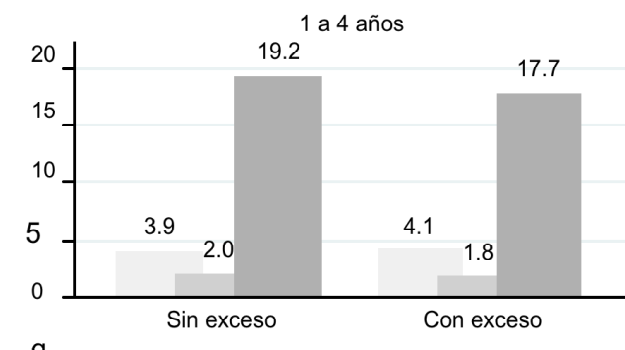

g

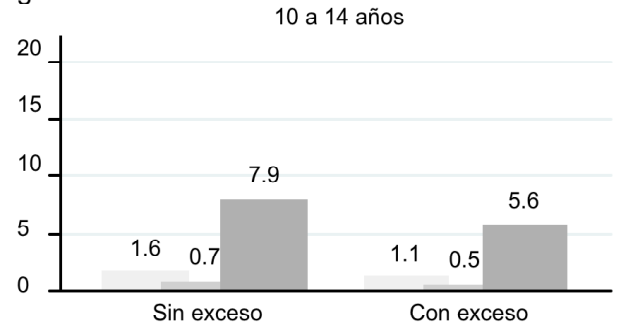

Sin exceso

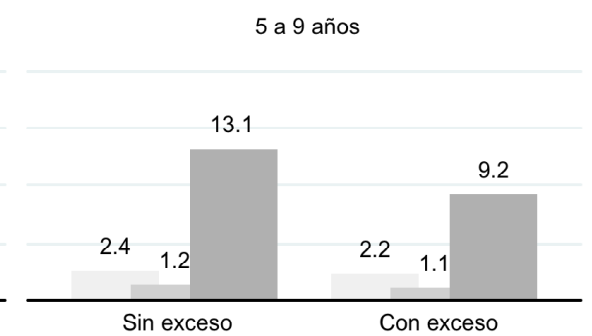

15 a 17 años

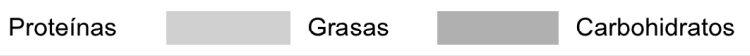

Sin exceso; IMC $<25$. Con exceso; IMC>=25. (Equivalentes de IMC según International Obesity Task Force) 
con su requerimiento medio de energía total/día (25).

En Colombia la lactancia exclusiva es baja, sólo un $6,0 \%$ de los menores a los seis meses es lactado de manera exclusiva y el uso del biberón en este grupo es un 45\%, y 63\% entre los 6 y 9 meses (3). En los menores de 2 años que reciben lactancia materna y en los lactados de manera exclusiva, el VCT derivado de proteínas es un 5\%, mientras que en los que reciben alimentación complementaria, y sucedáneos de la leche materna, incluida leche entera de vaca, este puede llegar al 15\%-20\% (26), el aquí reportado es el doble, 10,4\%. Dada la evidencia de que el excesivo consumo de proteínas en los dos primeros años de vida está asociado al exceso de peso y la obesidad en la infancia tardía, se ha propuesto limitar la cantidad de proteínas en las fórmulas infantiles para el primer año y limitar el consumo de leche de vaca en el segundo (25-26). En países de bajo ingreso la evidencia sobre la ingesta alta de proteínas, el incremento del peso y el riesgo tardío de enfermedades crónicas es limitada, en Guatemala las cifras de VCT derivado de proteínas en los dos primeros años de edad, son similares a las aquí reportadas (6). Sin embargo, en Guatemala como en Colombia dado que aún persiste la desnutrición, el discurso sobre el exceso proteínas en los primeros dos años pareciera no tener relevancia, desconociendo que en la desnutrición la calidad de la proteína es otro determinante del aumento del riesgo de enfermedad crónica en la edad adulta (6). De otra parte, dietas altas en proteínas son utilizadas con éxito en el tratamiento de la obesidad en niños y adultos, donde las metas terapéuticas son la reducción de la circunferencia abdominal, la fracción LDL del colesterol, la presión sanguínea y la regulación de la glucosa sérica $(8,27)$. Lo anterior corrobora nuestros hallazgos, en el sentido de que la composición de la dieta, juega un rol importante en la génesis de la obesidad, como también en su tratamiento.

Es importante resaltar que el VCT derivado de proteínas aumenta con el nivel socioeconómico, pero que en el nivel más bajo ya es excesivo. En todos los niveles de la escala socioeco- nómica este aumento se correlaciona débilmente con el VCT derivado de grasas y ocurre a expensas de VCT derivado de carbohidratos, lo que constituye una paradoja para el exceso de peso en los niños estudiados, el exceso está asociado al elevado consumo de proteínas aún en la pobreza. Pero el asunto es mucho más complejo, los patrones de consumo de alimentos en la pobreza cargan dinámicas metabólicas que como un todo favorecen el exceso. Se ha reportado que el consumo de leche entera de vaca se constituye en la principal fuente proteica en niños y por lo tanto, determinante del excesivo consumo de proteínas y grasa en los menores de dos años $(6,26)$. Ni la cantidad de bebidas tipo kola, ni la de bebidas azucaradas y tipo gaseosa, aunque similares a la cantidad consumida de leche de vaca, son diferentes entre sujetos con exceso y no, aspecto ya evidenciado en niños colombianos y en la ENSIN-2010 $(4,5)$. Aquí también reportamos seis alimentos cuya cantidad de consumo se asoció de manera inversa al riesgo de exceso, éstos hacen parte de un patrón previamente identificado y denominado "tradicional/almidón", que ha sido reportado como protector en Colombia y países de ingresos bajos y medios (4).

La organización Mundial de la Salud (OMS), dado lo complejo que es prevenir la obesidad en niños, ha propuesto intervenciones multifactoriales y en diferentes niveles con énfasis en el ambiente escolar (28). Sin embargo en Colombia como en muchos países de la región, la política pública dadas las limitaciones de datos locales, se ha construido con la evidencia de otros contextos y centrado sólo en dos variables de este complejo causal, la actividad física en el nivel individual y en la alimentación a nivel grupal, centrándose en el aumento del consumo de frutas y verduras, el desestimulo al consumo de bebidas azucaradas, tipo kola, gaseosa y los alimentos bocadillo, y de manera más general e inespecífica en la adopción de estilos de vida "saludables", algunas estrategias proponen incluso reemplazar el consumo de bebidas azucaradas y tipo gaseosa por leche (29). Si bien los resultados de

\section{TABLA 4}

Cantidades de alimentos (g) y asociación con el exceso de peso en menores de 18 años sin embarazo. Colombia, 2009-2011.

\begin{tabular}{|c|c|c|c|c|c|c|}
\hline \multirow[t]{2}{*}{ Alimento } & \multirow{2}{*}{$\begin{array}{c}n \\
{[430]}\end{array}$} & \multicolumn{2}{|c|}{ Promedio $\pm \mathrm{EE}^{\mathrm{a}}$} & \multirow[t]{2}{*}{$\mathrm{p}^{c}$} & \multirow{2}{*}{$\begin{array}{l}\text { Diferencia ajustada } \\
\qquad(I C ; 95 \%)^{d}\end{array}$} & \multirow[t]{2}{*}{$p^{e}$} \\
\hline & & Normal $^{b}$ & Exceso $^{b}$ & & & \\
\hline Leche vaca hervida & 157 & $224(15)$ & $405(41)$ & $<0,0001$ & 180 (77 a 284) & 0,001 \\
\hline Gaseosas y bebidas Azucaradas & 114 & $277(35)$ & $471(87)$ & 0,042 & $6(-113$ a 115) & 0,914 \\
\hline Lenteja Grano Entero & 63 & $50(5)$ & $49(12)$ & 0,932 & $-37(-59 a-15)$ & 0,002 \\
\hline Agua de Panela & 46 & $317(30)$ & $180(115)$ & 0,252 & $-105(-197 \mathrm{a}-14)$ & 0,026 \\
\hline Leche entera Polvo & 38 & $11(2)$ & $29(5)$ & $<0,0001$ & $24(10$ a 37$)$ & 0,001 \\
\hline Gaseosa tipo Kola & 30 & $222(25)$ & $296(56)$ & 0,244 & $-36(-244$ a 172$)$ & 0,704 \\
\hline Avena Grano Entero & 27 & $13(2)$ & $7(4)$ & 0,262 & $-7(-14 \mathrm{a}-0)$ & 0,041 \\
\hline Jugo de naranja & 18 & $54(9)$ & $96(20)$ & 0,079 & $78(48$ a 108) & 0,001 \\
\hline Gelatina & 18 & $189(21)$ & $170(47)$ & 0,716 & $-139(-361 \mathrm{a}-38)$ & 0,030 \\
\hline Envuelto de mazorca & 10 & $75(14)$ & $35(41)$ & 0,382 & $-76(-99 a-52)$ & 0,002 \\
\hline Salchichón & 11 & $63(13)$ & $23(27)$ & 0,211 & $-41(-74 a-8)$ & 0,026 \\
\hline Otra leche en polvo & 7 & $4(1)$ & $27(3)$ & 0,036 & $28(7$ a 48$)$ & 0,036 \\
\hline
\end{tabular}

a Promedio (g) del tamaño de la porción y su error estándar (EE).

b Sin exceso; IMC $<25$. Con exceso; >=25. Equivalentes de IMC según International Obesity Task Force (IOFT) (14)

c Valor de p para el test de diferencia de promedios entre niños con y sin exceso de peso (t de student).

d Las diferencias fueron ajustadas con base en un modelo de regresión lineal con el tamaño de la porción de un alimento especifico como variable dependiente y el exceso de peso (binomial), el sexo, la edad (continúa) y el nivel socioeconómico (SISBEN).

e Valor de p para el test de diferencia de promedios ajustados entre niños con y sin exceso de peso (t de student). 
estas estrategias "mejoran" los niveles de actividad física o de hábitos "saludables", no es clara su utilidad en la prevención del exceso de peso, y a menudo se confunde el resultado de la prevención con el del tratamiento de los sujetos ya con exceso. En escolares estadounidenses en el período 2001-2009 a pesar de la mejoría en los niveles de actividad física, del aumento del consumo de frutas y verduras y del descenso del consumo de bebidas azucaradas, el exceso de peso y la obesidad siguieron en aumento y sólo después de cuatro años la prevalencia del exceso tendió a estabilizarse (30). En una intervención mucho más compleja realizada durante dos años en varios niveles y al menos cinco variables incluidas la actividad física y la dieta en escolares en México, si bien se mejoró el entorno escolar, no se obtuvieron resultados sobre el exceso de peso (12), lo que demuestra el limitado impacto de estas intervenciones para prevenir o tratar el exceso de peso, incluso cuando se siguen las directrices de OMS (30).

Los resultados aquí presentados corroboran, al menos para el caso colombiano, que la transición alimentaria recientemente descrita con base en datos de la ENSIN-2010 (4), pueden sustentar cambios en las intervenciones y políticas hasta ahora basadas en otros contextos, de lo contrario éstas tendrán impacto limitado. De acuerdo con la evidencia aquí presentada una meta deseable para prevenir el exceso de peso desde la dieta de los niños colombianos es la recomposición del VCT, disminuir la cantidad de proteínas hasta lograr un VCT derivado de ellas inferior al 10\% en todas las edades y a expensas del aumento en el VCT derivado de carbohidratos, con el aumento de los complejos y la fibra dietaria.

La principal limitación que tiene este estudio es la dificultad para comparar los resultados con otros de países del área, que no cuentan datos del consumo dietario en niños. Otra limitación es que los datos provienen de un estudio de tipo transversal y no es posible establecer relaciones causales con el exceso de peso y finalmente, debido a las limitaciones de las tablas de composición de alimentos, no fue posible descomponer la ingesta de proteínas para conocer cuánta es origen animal o vegetal. Sin embargo, como fortalezas deben mencionarse que surge de un estudio de dieta que aplico $\mathrm{R} 24 \mathrm{H}$ repetidos, en los que se incorporó la más alta tecnología disponible en Colombia y que logró establecer la dieta usual en vez de la actual, que se evaluaron todos los posibles confusores de las relaciones descritas, incluida la actividad física y la ingesta total de energía/día, dos de las principales falencias en otros estudios sobre consumo.

En conclusión, establecimos que el exceso de peso en niños colombianos está asociado a la composición de la dieta (VCT), en particular con la energía derivada del consumo de proteínas a expensas del consumo de carbohidratos. También que el exceso de consumo de proteínas ocurre incluso en el nivel más bajo de la escala socioeconómica y está asociado a las cantidades/día de la ingesta de leche. Como ya se había advertido para niños colombianos en una muestra independiente de ésta, ni la cantidad consumida de bebidas azucaradas o tipo gaseosa están asociadas al exceso de peso. Sin embargo, las cantidades consumidas son altas y similares a las de la leche de vaca. Además, que los alimentos constitutivos de lo que se ha identificado en Colombia como el patrón "tradicional/ almidón", aparentemente son protectores del exceso de peso (4). Estos hallazgos se constituyen en otra pieza de un rompecabezas complejo de armar, que apunta a la comprensión del exceso de peso en niños colombianos, para incorporarla junto con otras a la política pública en el propósito de prevenir el exceso de peso infantil y sus consecuencias relacionadas con la enfermedades no transmisibles en la infancia tardía y la edad adulta. La política pública y los mensajes basados en contextos europeos o americanos, si bien pueden mejorar los entornos escolares y familiares y llevar información valiosa a la comunidad sobre su autocuidado, sin un marco amplio y complementario con la realidad de la microrregión y nuestra cultura, pueden ser limitadas al no incorporar otras posibles causas del exceso de peso en niños particulares de nuestra historia y transición alimentaria y nutricional.

\section{RESUMEN}

El objetivo fue describir el valor calórico total (VCT) derivado de macronutrientes en menores de 18 años con relación al exceso de peso, en cuatro ciudades de Colombia, 2009-2011. Se realizaron dos recordatorios del consumo dietario en las últimas 24 horas aplicados a 505 niños entre 0 y 17 años, el 51\% residentes en áreas urbanas. Además, se estableció su estado de nutrición con base en antropometría. Mediante regresión lineal se estableció la asociación del VCT y el exceso de peso. El sobrepeso en niños sin retraso en talla fue de $6,2 \%$. El exceso de peso (IMC>=25) fue de $16,3 \%$, El exceso en la zona urbana fue de $18,2 \%$ y en la rural de $14,2 \%, p=0,253$. Los niños con exceso de peso consumieron usualmente $0,25 \mathrm{~g} / \mathrm{kg} /$ día más de proteínas (IC 95\%; 0,11 a $0,40),-0,18 \mathrm{~g} / \mathrm{kg} /$ día menos de grasa (IC 95\%; -0,25 a -0,11) y $-1,80 \mathrm{~g} / \mathrm{kg} /$ día menos de carbohidratos (IC 95\%; -2,50 a -1,10), que aquellos sin exceso de peso, para todas las diferencias $\mathrm{p}<$ 0,0001 . Los sujetos con exceso de peso consumieron $0,8 \%$ más VCT derivado de proteínas que aquellos sin exceso, $p=0,006$. El exceso de peso en niños colombianos se asoció al VCT, en particular con la energía derivada del consumo de proteínas y también se asoció a las cantidades/día de la ingesta de leche.

Palabras clave: Niños; obesidad infantil; ingestión de energía; dieta; Colombia.

Conflicto de interés: Los autores declaran que no tienen conflicto de interés de ningún tipo, ni real o potencial sobre los resultados presentados.

Financiamiento: Este estudio fue posible por la cofinanciación del Departamento Administrativo de Ciencia, Tecnología e Innovación de Colombia (COLCIENCIAS), código; 110245921548 y de la Secretaria Distrital de Salud de Bogotá, la Gobernación de Cundinamarca, y las Universidades Nacional de Colombia e Industrial de Santander. Código interno UIS; 8677.

\section{REFERENCIAS}

1. Rahman SA, Adjeroh D. SurfaceBased Body Shape Index and Its Relationship with All-Cause Mortality. PLOS ONE. 2015; 10:1-21.

2. Popkin BM, Adair LS, Ng SW. NOW AND THEN: The Global Nutrition Transition: The Pandemic of Obesity in Developing Countries. Nutr Rev. 2012; 70:3-21.

3. Instituto Colombiano de Bienestar Familiar. Encuesta Nacional de la Situación Nutricional en Colombia, ENSIN-2010. Disponible en: http://www.icbf.gov.co/portal/ page/portal/Portal/CBF/NormatividadGestion/ENSIN1/ ENSIN2010

4. Ocampo PR, Prada GE, Herrán OF. Food consumption patterns and excess childhood weight; a survey of the nutritional situation in Colombia, 2010. Rev Chil Nutr. 2014; 41:351-59.

5. McDonald CM, Baylin A, Arsenault JE, Mora-Plazas M, 
Villamor E. Overweight is more prevalent than stunting and is associated with socioeconomic status, maternal obesity, and a snacking dietary pattern in school children from Bogotá, Colombia. J Nutr. 2009; 139:370-76

6. Michaelsen KF, Larnkjær A, Mølgaard C. Amount and quality of dietary proteins during the first two years of life in relation to NCD risk in adulthood. Nutr Metab CardiovasC Dis. 2012; 22:781-86

7. Gutiérrez CL, Vásquez-Garibay E, Romero-Velarde E, TroyoSanromán R, Cabrera-Pirava C, Magaña OR. Consumption of sweetened beverages as a risk factor for adolescent obesity in Guadalajara, Mexico. Bol Med Hosp Infant Mex. 2009; 66:522-28.

8. Boisvert JA, Harrel A. Integrative Treatment of Pediatric Obesity: Psychological and Spiritual Considerations. JIM. 2015; 14:40-7.

9. Monteiro CA, Levy RB, Claro RM, Ribeiro IR, Cannon G. Increasing consumption of ultra-processed foods and likely impact on human health: evidence from Brazil. Public Health Nutr. 2010; 14:5-13.

10. Richard Tiffin $R$, Salois M. Inequalities in diet and nutrition. Nutr Soc. 2012; 71:105-11.

11. Poletti OH, Barrios L. Overweight, obesity, eating habits, physical activity and leisure time use in schoolchildren in Corrientes (Argentina). Rev. Cubana. Pediatr. 2007; 79:[on line].

12. Safdie $M$, Jennings-Aburto $N$, Lévesque $L$, Ian Janssen I, Campirano-Núñez F, López-Olmedo $N$, et al. Impact of a school-based intervention program on obesity risk factors in Mexican children. Salud Publica Mex. 2013; 55(suppl 3):S374-S87.

13. Willet WC. Nutritional epidemiology. Third edition. Oxford University Press. New York, 2013.

14. Cole TJ, Bellizzi MC, Flegal KM, Dietz WH. Establishing a standard definition for child overweight and obesity worldwide: international survey. BMJ. 2000; 320:1-6.

15. Lauritsen J. FoodCalc v. 1,3. Diet, cancer and health project. Danish: Danish Cancer Society; 1998.

16. lowa State University. PC-Side, version 1.02. lowa: Department of Statistics and Center for Agricultural and Rural Development; 2004.

17. Jiménez AZ, Prada GE, Herrán OF. Scales to measure food security in Colombia. Are they valid? Rev Chil Nutr. 2012; 39:8-17.
18. Stata Statistical Software: Release 13.1. Collegue Station, TX: StataCorp LP.

19. Instituto Nacional de Salud Pública. National health and nutrition survey 2006 (ENSANUT). Cuernavaca, Morelos, México, 2008.

20. Kovalskys I, Bay L, Herscovici CR, Berner E. Prevalence of obesity in a population of 10-19 year olds in a pediatric center. (Argentina). Rev Chil Pediatr. 2005; 76:324-25.

21. Ogden $C L$, Carrol MD, Kit BK, Flegal KM. Prevalence de obesity and trends in body mass index among US children and adolescents, 199-2010. JAMA. 2012; 307:483-90.

22. de Assis MA, Rolland-Cachera MF, Grosseman S, de Vasconcelos FA, Luna ME, Calvo MC, et al. Obesity, overweight and thinness in schoolchildren of the city of Florianopolis, Southern Brazil. Eur J Clin Nutr. 2005; 59:1015-21.

23. Vio F, Albala C, Kain J. Nutrition transition in Chile revisited: mid-term evaluation of obesity goals for the period 2000-2010. Public Health Nutr. 2008; 11:405-12.

24. Pysz K, Leszczyńska T, Aneta Kopeć A. Anthropometric assessment of the nutritional status of children and adolescents residing in selected polish orphanages based on their energy intake and physical activity level. Rocz Panstw Zakl Hig. 2015; 66:77-83

25. Pysz1 M, Leszczyńska T, Cieślik E, Kopeć A, Wielgos B, Piątkowska E. Relationship between the intake of energy and basic nutrients and the BM/ values in group of children aged 10-12. Rocz Panstw Zakl Hig. 2014; 65:345-52.

26. Michaelsen KF, Greer FR. Protein needs early in life and long-term health. Am J Clin Nutr. 2014; 99(3):718S-22S

27. Damsgaard CT, Papadaki A, Jensen SM, Ritz C, DalsKov $S M$, Hlavaty $P$, et al. Higer protein diets consumed ad libitum improve cardiovascular risk markers in children of overweight parents from eight European countries. J Nutr. 2013; 143:810-17.

28. OMS. School policy framework; implementation of the WHO global strategy on diet, physical activity and health. WHO. Geneva, 2008.

29. Albala C, Ebbeling CB, Cifuentes M, Lera L, Bustos $N$, and Ludwig DS. Effects of replacing the habitual consumption of sugar-sweetened beverages with milk in Chilean children. Am J Clin Nutr. 2008; 88:605-11.

30. Lannotti RJ, Jing Wang J. Trends in Physical Activity, Sedentary Behavior, Diet, and BMI Among US Adolescents, 2001-2009. Pediatrics. 2013; 132:606-14. 